\title{
An Extremum Problem for Convex Polygons
}

\section{By \\ Gerhard Larcher and Friedrich Pillichshammer}

(Vorgelegt in der Sitzung der math.-nat. Klasse am 14. Dezember 2006 durch das w. M. August Florian)

\begin{abstract}
In the Euclidean plane, consider a convex $n$-gon with unit perimeter. For a certain class of functions $f:[0,1 / 2] \rightarrow \mathbb{R}_{0}^{+}$we establish the least upper bound on the sum of the values of $f$ over the distances of all pairs of vertices of the polygon.
\end{abstract}

Mathematics Subject Classification (2000): 52A40, 51M04, 51K05.

Key words: Convex polygon, Euclidean distance, sum of distances.

Let $f:[0,1 / 2] \rightarrow \mathbb{R}_{0}^{+}$be a function. For $n \geq 3$, let $x_{1}, \ldots, x_{n}$ be the (pairwise different) vertices of a convex polygon with unit perimeter in the Euclidean plane. Define

$$
S_{n}(f):=\sum_{1 \leq i<j \leq n} f\left(\left\|x_{i}-x_{j}\right\|\right)
$$

where $\|\cdot\|$ denotes the Euclidean norm.

We ask, what is the least upper bound on $S_{n}(f)$ ?

For the specific function $f(x)=x$ this question was stated as open problem in [1] and completely solved in [2, Theorem 1] (here even a best possible lower bound was given). Furthermore, in [2, Theorem 3] an upper bound was given (which is best possible for even $n$, but not for odd $n$ ) if $f(x)=x^{2}$. 
In this short note we give the solution to this question for a certain class of functions. Our result generalizes [2, Theorem 1] and gives the answer to [2, Open Problem 2].

Theorem 1. Let $f:[0,1 / 2] \rightarrow \mathbb{R}_{0}^{+}$be such that the function $x \mapsto f(x) / x$ attains its maximum in $x=1 / 2$. Then for any $n \geq 3$ and any convex polygon with $n$ vertices and with unit perimeter in the Euclidean plane we have

$$
S_{n}(f) \leq f\left(\frac{1}{2}\right)\left\lfloor\frac{n}{2}\right\rfloor\left\lceil\frac{n}{2}\right\rceil .
$$

Furthermore the bound is approached arbitrarily closely by a convex polygon with vertices $x_{1}, \ldots, x_{|n / 2|}$ that are arbitrarily close to the origin and $x_{|n / 2|+1}, \ldots, x_{n}$ that are arbitrarily close to the point $1 / 2$ on the $x$-axis. If the function $x \mapsto f(x) / x$ attains its maximum if and only if $x=1 / 2$ and if $n \geq 4$, then the above inequality is even strict.

Remark 1. Note that it is not enough that only $f$ attains its maximum in $x=1 / 2$. For example consider the function $f(x)=\sqrt{x}$. If $n=6 m$ points $x_{1}, \ldots, x_{6 m}$ are distributed evenly among the vertices of a regular triangle of edge-length $1 / 3$, then we have $S_{6 m}(\sqrt{\cdot})=$ $\Sigma_{1 \leq i<j \leq 6 m} \sqrt{\left\|x_{i}-x_{j}\right\|}=12 \mathrm{~m}^{2} / \sqrt{3}>9 \mathrm{~m}^{2} / \sqrt{2}=\sqrt{1 / 2}\lfloor 6 \mathrm{~m} / 2\rfloor\lceil 6 \mathrm{~m} / 2\rceil$, such that the bound from Theorem 1 is not valid any more.

For the proof of Theorem 1 we need the following elementary lemma.

Lemma 1. Let $f:[0,1 / 2] \rightarrow \mathbb{R}_{0}^{+}$be such that the function $x \mapsto f(x) / x$ attains its maximum in $x=1 / 2$. Let $n \geq 3$ and let $a_{1}, \ldots, a_{n}$ be the side-lengths of a plane convex $n$-gon with perimeter at most one, i.e., $\sum_{k=1}^{n} a_{n} \leq 1$. Then we have

$$
\sum_{k=1}^{n} f\left(a_{k}\right) \leq 2 f\left(\frac{1}{2}\right) .
$$

Proof. Trivially we have $a_{k} \leq 1 / 2$ for all $k=1, \ldots, n$ and therefore

$$
\sum_{k=1}^{n} f\left(a_{k}\right)=\sum_{k=1}^{n} \frac{f\left(a_{k}\right)}{a_{k}} a_{k} \leq 2 f\left(\frac{1}{2}\right) \sum_{k=1}^{n} a_{k} \leq 2 f\left(\frac{1}{2}\right) .
$$

Now we give the proof of Theorem 1 .

Proof of Theorem 1. We use the ideas from [2, Proof of Theorem 1]. Let the vertices $x_{1}, \ldots, x_{n}$ of the polygon $P$ be arranged clockwise. 
Assume first that $n$ is even. Now we consider the $\left(\begin{array}{c}n / 2 \\ 2\end{array}\right)$ convex quadrangles

$$
Q_{i, j}:=\overline{x_{i} x_{j} x_{i+\frac{n}{2}} x_{j+\frac{n}{2}} x_{i}}
$$

for all $i$ and $j$ satisfying $1 \leq i<j \leq \frac{n}{2}$. Let

$$
\begin{aligned}
u(i, j):= & f\left(\left\|x_{i}-x_{j}\right\|\right)+f\left(\left\|x_{j}-x_{i+\frac{n}{2}}\right\|\right)+f\left(\left\|x_{i+\frac{n}{2}}-x_{j+\frac{n}{2}}\right\|\right) \\
& +f\left(\left\|x_{j+\frac{n}{2}}-x_{i}\right\|\right) .
\end{aligned}
$$

As $Q_{i, j}$ is convex and contained in $P$ it follows from Lemma 1 that $u(i, j) \leq 2 f(1 / 2)$. Trivially $\left\|x_{i}-x_{i+n / 2}\right\| \leq \frac{1}{2}$ for all $i$ such that $1 \leq i \leq \frac{n}{2}$ and $\left\|x_{i}-x_{i+n / 2}\right\|<\frac{1}{2}$ for at least one such choice of $i$. Since also $f$ attains its maximum in $x=1 / 2$ we have

$$
\begin{aligned}
S_{n}(f)= & \sum_{1 \leq i<j \leq \frac{n}{2}} u(i, j)+\sum_{i=1}^{\frac{n}{2}} f\left(\left\|x_{i}-x_{i+\frac{n}{2}}\right\|\right) \leq\left(\begin{array}{c}
\frac{n}{2} \\
2
\end{array}\right) 2 f\left(\frac{1}{2}\right) \\
& +\frac{n}{2} f\left(\frac{1}{2}\right)=f\left(\frac{1}{2}\right) \frac{n^{2}}{4} .
\end{aligned}
$$

Here the first equality can be easily checked by counting all the different distances occurring on the right-hand side. It is clear that each distance on the right side of the equality appears at most once, but on the other hand we sum up $4\left(\begin{array}{c}n / 2 \\ 2\end{array}\right)+\frac{n}{2}=\left(\begin{array}{c}n \\ 2\end{array}\right)$ distances, so the equality is true. Furthermore, if $f(x) / x<2 f(1 / 2)$ for all $x \in[0,1 / 2)$, then also $f(x)<f(1 / 2)$ for all $x \in[0,1 / 2)$ and the above inequality is strict as well.

Now let $n$ be odd. In this case we consider the $\left(\begin{array}{c}\frac{n-1}{2} \\ 2\end{array}\right)$ convex quadrangles

$$
Q_{i, j}:=\overline{x_{i} x_{j} x_{i+\frac{n+1}{2}} x_{j+\frac{n+1}{2}} x_{i}}
$$

for $1 \leq i<j \leq \frac{n-1}{2}$ and the $\frac{n-1}{2}$ convex triangles

$$
R_{i}:=\overline{x_{i} x_{\frac{n+1}{2}} x_{i+\frac{n+1}{2}} x_{i}}
$$

for $1 \leq i \leq \frac{n-1}{2}$. Let

$$
\begin{aligned}
u(i, j):= & f\left(\left\|x_{i}-x_{j}\right\|\right)+f\left(\left\|x_{j}-x_{i+\frac{n+1}{2}}\right\|\right)+f\left(\left\|x_{i+\frac{n+1}{2}}-x_{j+\frac{n+1}{2}}\right\|\right) \\
& +f\left(\left\|x_{j+\frac{n+1}{2}}-x_{i}\right\|\right)
\end{aligned}
$$

and

$$
v(i):=f\left(\left\|x_{i}-x_{\frac{n+1}{2}}\right\|\right)+f\left(\left\|x_{\frac{n+1}{2}}-x_{i+\frac{n+1}{2}}\right\|\right)+f\left(\left\|x_{i+\frac{n+1}{2}}-x_{i}\right\|\right) .
$$


As $Q_{i, j}$ and $R_{i}$ are both convex polygons and contained in $P$, it follows from Lemma 1 that $u(i, j) \leq 2 f(1 / 2)$ and $v(i) \leq 2 f(1 / 2)$. So

$$
\begin{aligned}
S_{n}(f) & =\sum_{1 \leq i<j \leq \frac{n-1}{2}} u(i, j)+\sum_{i=1}^{\frac{n-1}{2}} v(i) \leq 2 f\left(\frac{1}{2}\right)\left(\left(\begin{array}{c}
\frac{n-1}{2} \\
2
\end{array}\right)+\frac{n-1}{2}\right) \\
& =f\left(\frac{1}{2}\right) \frac{n^{2}-1}{4} .
\end{aligned}
$$

Here the first equality can be checked as above. If $n>3$, then we must have $v(i)<2 f(1 / 2)$ for at least one $i$ satisfying $1 \leq i \leq \frac{n-1}{2}$.

Hence the desired bound is proved in both cases.

It is easy to see that the bound is approached arbitrarily closely by the point distributions given in Theorem 1 . We just mention that $\lim _{x \rightarrow 0^{+}} f(x)=0$ since $0<f(x) / x \leq 2 f(1 / 2)$ for all $x \in(0,1 / 2]$.

\section{References}

[1] Audet, C., Hansen, P., Messine, F. (2007) Extremal problems for convex polygons. J. Global Optim. (to appear)

[2] LARCHER, G., PillichShAmmer, F. (2007) The sum of distances between vertices of a convex polygon with unit perimeter. Amer. Math. Monthly (to appear)

Authors' address: Prof. Dr. Gerhard Larcher and Prof. Dr. Friedrich Pillichshammer, Institut für Finanzmathematik, Universität Linz, Altenberger Straße 69, 4040 Linz, Austria. E-Mail: gerhard.larcher@jku.at, friedrich.pillichshammer@ jku.at. 